\title{
RTOS based embedded controller implementation of a bilateral control system
}

\author{
A.M. Harsha S. Abeykoon* and M. Branesh Pillai \\ Department of Electrical Engineering, Faculty of Engineering, University of Moratuwa, Katubedda, Moratuwa.
}

Revised: 30 January 2014; Accepted: 21 February 2014

\begin{abstract}
Bilateral tele-operation involves realization of the law of action and reaction between the human and the remote environment. In this research, an embedded system based bilateral control was developed on a microcontroller based motor driver platform. Bilateral tele-operation needs real-time representation of the forces and positions. This challenge was accomplished by incorporating a real-time operating system (RTOS) with a resource limited microcontroller. Sensorless torque detection technique was used to find the external torque while disturbance observer was used as a disturbance rejection tool and also to improve the system's robustness. When the motion controller was implemented on a microcontroller, 0 - $30 \mathrm{~Hz}$ frequency of force bandwidth could be achieved. In an ideal bilateral control, force/position errors should be zero. The experimental results in contact motion showed a force error less than $4 \%$ and a position error less than $6 \%$, which validates the proposal. This was a significant achievement as the bilateral controllers have never been successfully implemented using a microcontroller.
\end{abstract}

Keywords: Bilateral control, disturbance observer, haptics, microcontroller, reaction torque observer, real time operating system.

\section{INTRODUCTION}

Well before the dawn of the modern age, information was recorded in a pictorial language consistent with the simple life style. The next significant development was the storage methods for sound information. This was followed by the development of visual recording technology, especially after the invention of the television. In the modern world, communication technologies have grown rapidly with the boom in the Internet enabled transmission of information including text, sound and video files worldwide. Although audio and visual forms perceived by humans can be stored and reproduced in a remote place, the senses of nose (smell) and tongue (taste) cannot normally be transmitted nor stored in electronic means.

This paper focuses on the transmission of sense of touch (haptic) information by electronic means from a remote point. This technology has been developed specially by many researchers of the Ohnishi Laboratory of the Keio University, Japan (Nishimura et al., 2006; Abeykoon \& Ohnishi, 2007; 2008).

Most of the controllers in our day-to-day applications are unilateral. As the name implies, the user's intention travels unilaterally to the actuator. A simple example would be a TV remote controller where the human presses a channel number and the control signal travels unilaterally from the TV controller to the TV set, where the channel is changed. Four senses of the human, namely smell, vision, hearing and taste can be considered as unilateral and the sensing is done unilaterally as shown in Figure 1. However, the sense of touch is not unilateral. It obeys the law of action and reaction and the concept is shown in Figure 2.

Haptic sensation is a realization of the law of action and reaction (Katsura et al., 2007) between the human and the environment. If the sense of touch has to be transmitted to a remote place, then the reaction force from the environment should be felt by the operator in real-time. Therefore there should be a speedy way of communication between the two points once the object and the operator are kept away. There should be two identical manipulators at both ends: to sense the environment's reaction and to transmit it back to the operator; and to transmit the operator's intention to the environment 


\begin{tabular}{|l|l|l|l|}
\hline REAL WORLD & RECORDING & TRANSMISSION & UNI / BILATERAL \\
\hline & UNILATERAL \\
\hline
\end{tabular}

Figure 1: Transmission of sense of touch

(Figure 2). According to Figure 2, the transmission of real world haptic information communication between the operator and the real environment is in two directions, or bilateral.

If there is to be a successful solution for the transmission of haptic information then it should be bilateral. The bilateral control is a realization of the law of action and reaction between the master system, which the operator manipulates and the slave system, which is in contact with the environment (Katsura, 2004; Shimono, 2007; Abeykoon, 2008).

In control systems unknown signals affect the performance; unmodelled or undefined signals, which are generated may contribute to the disturbance. Usually friction and parameter changes of the process over time, may create another type of disturbance in the system. But to increase the system robustness, to attain high accuracy, and to achieve fast response, compensation of the disturbance is very important. As explained above, bilateral control works based on the law of action and reaction. To realize bilateral control, force/position information should be exchanged between the remote points very fast (Katsura et al., 2007). Such systems are currently realized using very fast computer based processors having many resources (Katsura, 2004; Shimono, 2007; Abeykoon, 2008;).

Bilateral control based tele-operation has been proposed by Ohnishi et al. (1996), Katsura (2004) and many others. Some research groups at the Keio University (Ohnishi et al., 1994) have used disturbance observer (DOB) to enhance the system robustness.

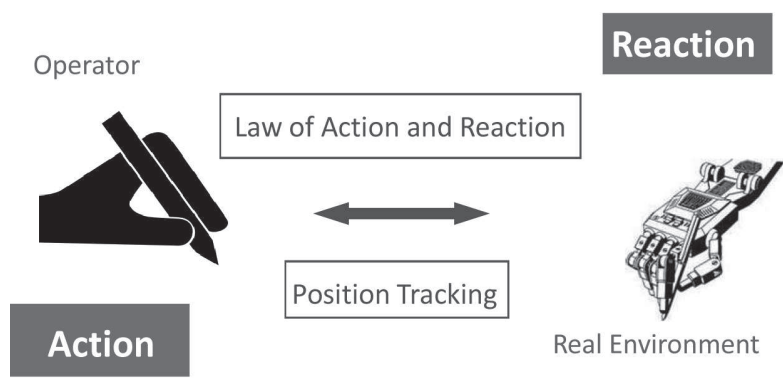

Figure 2: Transmission of real world haptic information

Reaction torque observer was experimentally verified by Katsura (2004) and Abeykoon (2008) and they have proved that it could be effectively used as a force/ torque sensor in DC motor based applications. In the above research they have used computer based systems with high-end processing capabilities, as the bilateral control requires near real-time representation of action and reaction. In this paper, the authors propose a novel embedded system based implementation of a bilateral tele-operation system. Microcontroller based RTOS has been used to accomplish the challenging time constraints (action and reaction). This was a significant achievement as the bilateral controllers have never been successfully implemented using a microcontroller.

Conventional bilateral control systems use force sensors. In this research, a reaction torque observer (RTOB) was used as the force sensor. A motor driver unit was also designed and developed. A feedback based current controller developed earlier (Abeykoon \& Senevirathne, 2012) has been used in this study.

\section{DISTURBANCE OBSERVER}

Disturbance observer is a tool for compensating disturbances, which is very useful and widely utilized in motion control applications of robotics. When applying a force to an object via a motor, we would expect the object to accelerate according to the Newton's second law as shown in the equation (1)

$\ddot{\theta}=\frac{\tau^{r e f}}{J}$

where,

$\ddot{\theta}$ - DC motor acceleration

$\tau^{r e f}$ - reference motor torque

$J$ - motor inertia 
When the motor is experiencing an external disturbance, the motor system can be represented by equation (2)

$$
\ddot{\theta}=\frac{\tau^{r e f}}{J}+\frac{\tau^{d i s}}{J}
$$

Where,

$$
\tau^{\text {dis }} \text { - disturbance torque }
$$

As the name suggests disturbance observer measures and estimates the disturbance, which is not normally measurable otherwise. Once the unknown disturbance is measured, it compensates for the disturbance. Its net effect on the system is a near "zero" disturbance through the effect of the compensation (Abeykoon \& Ohnishi, 2006).

\section{Modelling of disturbance observer}

A disturbance observer observes the disturbance force in the system without using any force sensors (Ohnishi et al., 1994;1996).

A servo motor in the joint space can be represented as follows,

$J \ddot{\theta}=T_{m}-T_{l}$

where;

$T_{m}$ - motor torque; $T_{l}-$ load torque; $J_{-}$motor inertia; $\ddot{\theta}$ - angular acceleration.

When a servo motor with one degree of freedom is considered, it could be represented as shown in Figure 3.

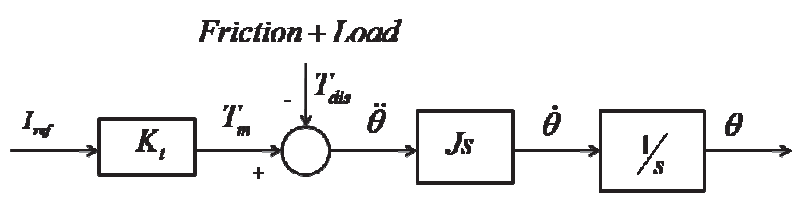

Figure 3: Motor with disturbance torque

The load torque is considered as,

$$
T_{l}=T_{\text {int }}+T_{e x t}+T_{f}+B \dot{\theta}
$$

$T_{\text {int }}$, the internal torque derived from the Lagrange motion equation consists of the inertia torque and the gravity effect. $T_{\text {ext }}$ consists of the torque external to the system. Friction term $T_{f}+B \dot{\theta}$ is the sum of Coulomb and viscosity terms; friction terms can be estimated as in Ohnishi et al. (1994).

Therefore for a DC servo motor, equation (3) could be rewritten as,

$$
J \ddot{\theta}=K_{t} I_{f}-\left(T_{\text {int }}+T_{e x t}+T_{f}+B \dot{\theta}\right)
$$

Equation (5) has two parameters, namely $J$ and torque constant $K_{t}$. Inertia can be changed due to the mechanical configuration of the system and the nominal inertia, which is available can be different from the manufacturer's given value.

$$
J=J_{n}+\Delta J
$$

Similarly parameter $K_{t}$ also changes,

$$
K_{t}=K_{t n}+\Delta K_{t}
$$

Here $J_{n}$ and $K_{t n}$ are nominal inertia and the nominal torque constant of the motor, respectively.

Disturbance torque $T_{d i s}$ is represented as

$$
T_{\text {dis }}=T_{l}+\Delta J \ddot{\theta}-\Delta K_{t} I_{r e f}
$$

By applying the value of $T_{l}$ in the above equation (8),

$T_{\text {dis }}=T_{\text {int }}+T_{\text {ext }}+T_{f}+B \dot{\theta}+\Delta J \ddot{\theta}-\Delta K_{t} I_{r e f} \ldots$

where,

$$
\begin{aligned}
& \Delta J=\left(J-J_{n}\right) \\
& \Delta K_{t}=\left(K_{t n}-K_{t}\right)
\end{aligned}
$$

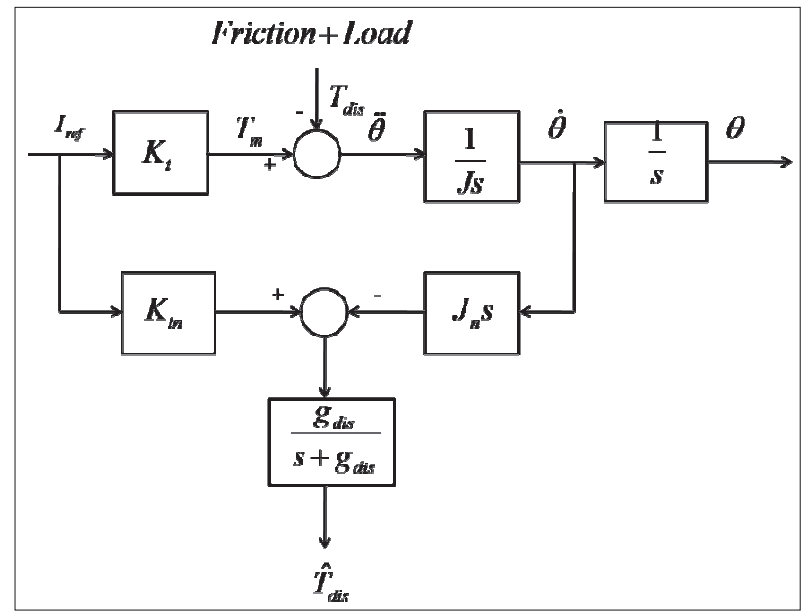

Figure 4: Block diagram of disturbance observer 
The block diagram of disturbance observer is shown in Figure 4.

Then the disturbance torque is estimated by the disturbance observer through the low-pass filter as equation (10).

$$
\hat{T}_{d i s}=\left(\frac{g_{d i s}}{s+g_{d i s}}\right) T_{d i s}
$$

Here $\hat{T}_{d i s}$ is the estimated disturbance torque and $g_{\text {dis }}$ is the observer gain. The disturbance observer calculates and estimates the reaction torque as quickly as possible (Ohishi et al., 1987; Sabanovic, 2003). By having the disturbance feedback it can compensate for the unknown disturbance acting on the system (Shimono et al., 2005; Senevirathne et al., 2012). If the frictional components are measured and eliminated from the disturbance output, the real reaction torque $T_{\text {rec }}$ can be measured. This is a variant of the disturbance observer, and it is called the reaction torque observer (RTOB).

Functional block diagram of the reaction torque observer is shown in Figure 5. This block diagram is similar to the disturbance observer except for the friction components.

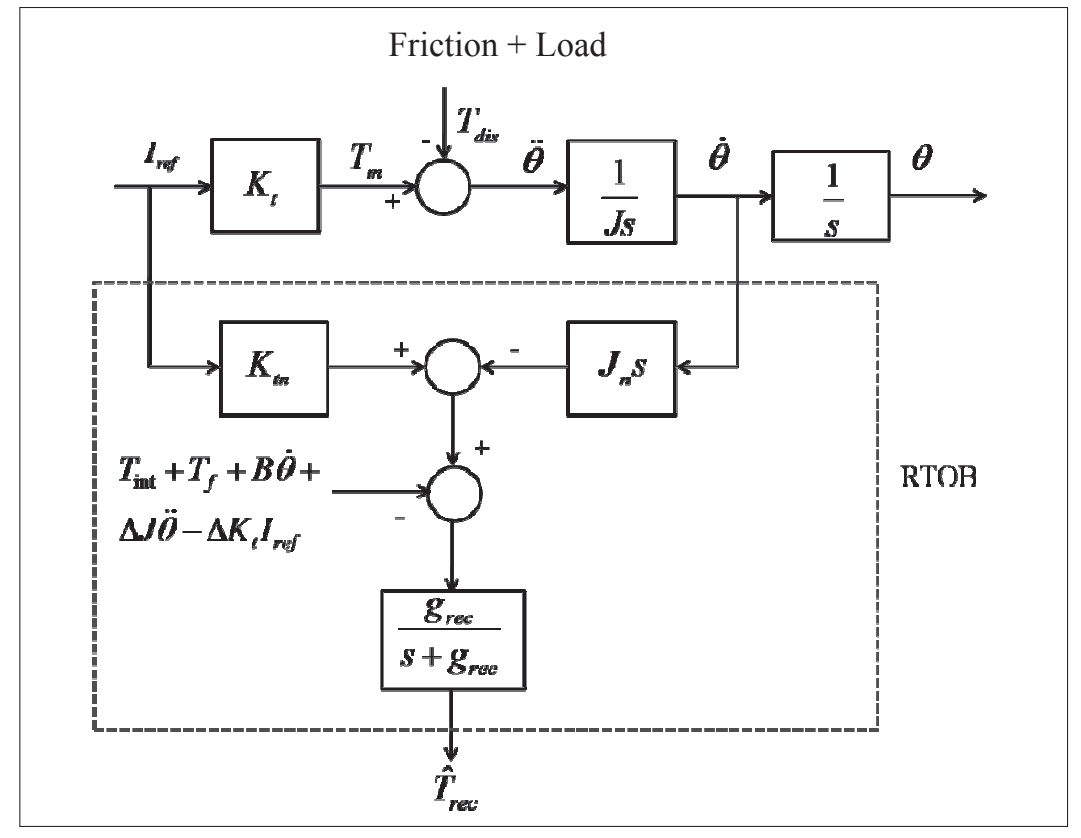

Figure 5: Block diagram of RTOB

The disturbance torque, which is estimated by the disturbance observer is depicted in equation (9) from which the friction components $T_{f}+B \dot{\theta}$ are subtracted (Ohnishi et al., 1994) and finally equation (11) can be derived.

$\widehat{T}_{r e c}=\frac{g_{r e c}}{s+g_{r e c}}\left(I_{r e f} K_{t n}-\Delta J \ddot{\theta}-\left(T_{d i s}-T_{e x t}\right)\right)$

Here $\hat{T}_{r e c}$ is the estimated reaction torque and $g_{\text {rec }}$ is the observer gain of reaction torque observer.

\section{BILATERAL CONTROL}

As the name suggests, bilateral controller controls the master and the slave sides from the responses of the slave and master sides, respectively (Sumiyoshi \& Ohnishi, 2004). These responses are converted as inputs to the master and slave sides. The slave side is controlled using the torque/position feedback of the master side and vice versa (Abeykoon \& Ohnishi, 2008) as shown in Figure 6.

A major objective of bilateral control is to achieve high transparency. In simple terms, following the literal meaning of the word "transparency", it means how well the torque/position information of the slave side is transparent to the master side (Yokokohji \& Yoshikawa, 1994). In other words, transparency is the match of the impedance perceived by the operator with the environment (Lawrence, 1993). 
As for control theory, it has been pointed out that the bilateral controllers cannot achieve transparency and stability simultaneously due to the uncertainties of the system and the environment (Katsura et al., 2008). Therefore, a bilateral system should strike a balance between the stability and the transparency (Hashtrudizaad \& Salcudean, 2002a;b). In conventional bilateral control, force sensors are used to detect the force of master and slave. For force control, many researchers use force sensors to detect external forces (George et al., 1983; Takeo \& Kosuge, 1997). Strain gauges are widely used as force sensors (Mayer et al., 2005) since strain demonstrates the external force. However, when it experiences motion with contact, usually the accuracy of the output deteriorates. Additionally, force sensors have narrow bandwidths. Therefore, the force sensation sensed through the force sensors is often dull (Katsura, 2004).

A force sensor can detect forces where the force sensor is located. In bilateral control applications, it is very difficult to fix the force sensor where the force should be sensed. Therefore, force sensors are often criticized, because they cannot produce a vivid sensation of the force (Katsura, 2004). Force sensor itself draws power from the system's measurand, which distorts the force to be measured. On the other hand, the disturbance observer is capable of providing a much wider bandwidth than the force sensors, because it is possible to set the sampling time short and set the observer gain high. Therefore, disturbance observer is more suitable for force measurement than force sensors. The bandwidth of the RTOB based force control was analyzed by Katsura et al., (2006). They have experimented with the frequency bandwidth of sensed force $(0-80 \mathrm{~Hz})$ successfully. However, in this research the authors have used a lower bandwidth $(0-30 \mathrm{~Hz})$ as the human interaction with a system usually involves lower frequencies. A wider bandwidth could not be obtained due to the resource constraints of the microcontroller.

Bilateral control is a realization of natural law of motion of two objects. But the input from the operator or the environmental response consists of elements of disturbance and not only the force. Therefore, to realize a good bilateral system, a good disturbance rejection mechanism should be in action. Robust motion control with bilateral control is achieved through the disturbance observer. Acceleration control plays an important role in realizing this. Bilateral control structure is derived based on the law of action and reaction (Katsura et al., 2008). When the operator manipulates the system from the master side, the slave side has to be in contact with the environment. However, in an application where the master and slave are different, scaling may be used.

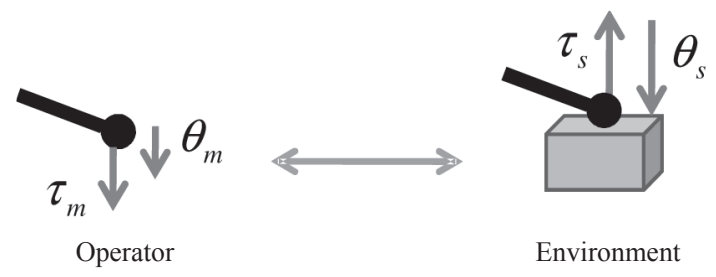

Figure 6: Basics of bilateral control

Figure 6 shows the basics of bilateral control. For bilateral control without scaling, the following equations could be derived.

$$
\begin{gathered}
\theta_{m}-\theta_{s}=0 \\
\tau_{m}+\tau_{s}=0
\end{gathered}
$$

Here subscript $m$ denotes the master and subscript $s$ denotes the slave. There are many scaling methods such as position, torque, impedance and time scaling (Itoh et al., 2000; Tsuji \& Ohnishi, 2004). In bilateral control it is necessary to attain both the ideal force control and the position control simultaneously. The equations (12) and (13) are transformed to (14) and (15) as accelerations.

$$
\begin{aligned}
& \ddot{\theta}_{m}-\ddot{\theta}_{s}=0 \\
& \ddot{\theta}_{m}+\ddot{\theta}_{s}=0
\end{aligned}
$$

Since disturbance observer is used as a force sensor in bilateral control, the sampling time should be small enough for a better response. The relationship of torque, which is represented as the acceleration is called the common mode between the master and the slave system, i.e. equation (15). Equation (14), which represents the position relationship is called as differential mode. This can be attributed to the sign of equations. Therefore, it is possible to realize both force control and position control independently in bilateral control (Shimono et al., 2005), when force and position are combined in the acceleration dimension. Equation (14) and (15) can be transformed into the following equations based on the optimal goal of bilateral control.

$$
\begin{aligned}
& \ddot{\theta}_{m}-\ddot{\theta}_{s}=\ddot{\theta}_{c o m} \rightarrow 0 \\
& \ddot{\theta}_{m}+\ddot{\theta}_{s}=\ddot{\theta}_{d i f} \rightarrow 0
\end{aligned}
$$

Disturbance observer and reaction torque observer are used in both master and slave sides as well. Equations (12) to (17) denote the basics of the bilateral control. DOB, RTOB and the basics of bilateral control could be represented as shown in Figure 7. 


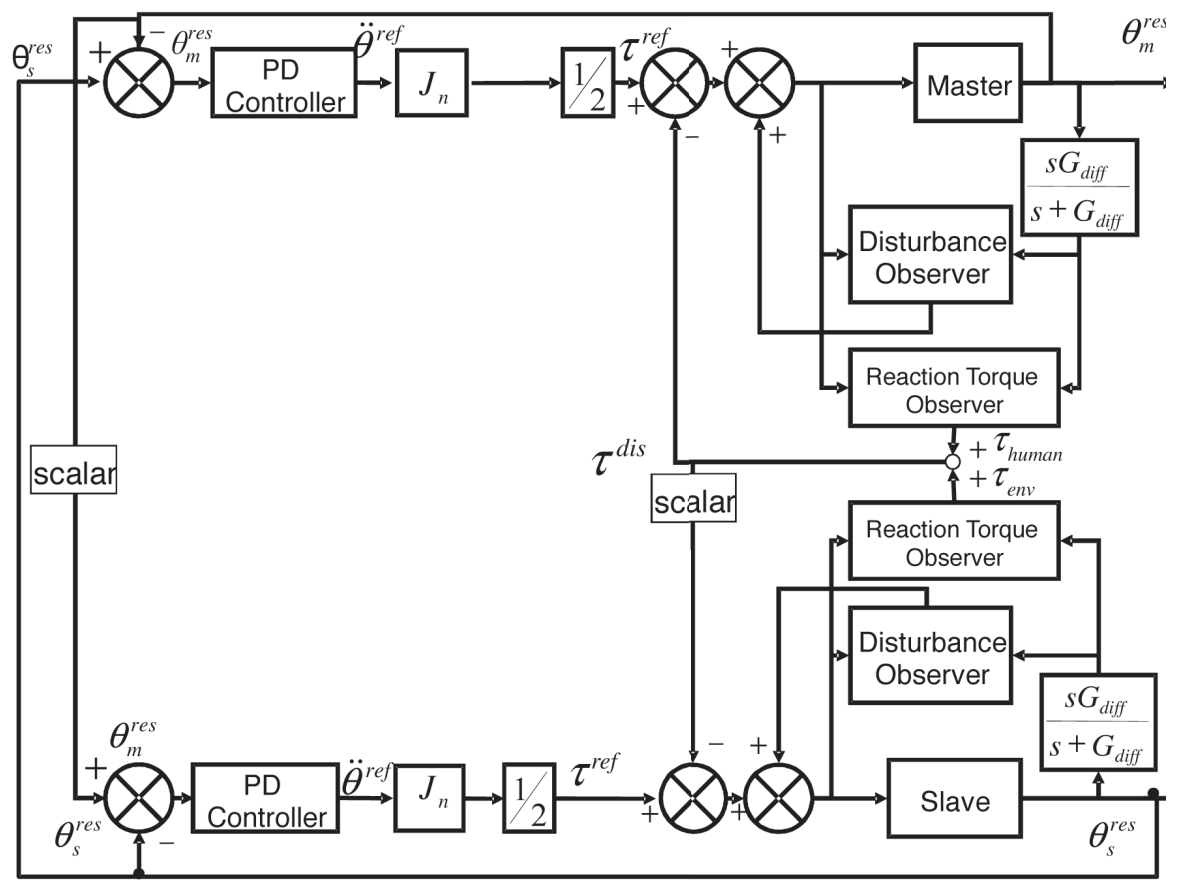

Figure 7: Block diagram of conventional bilateral control

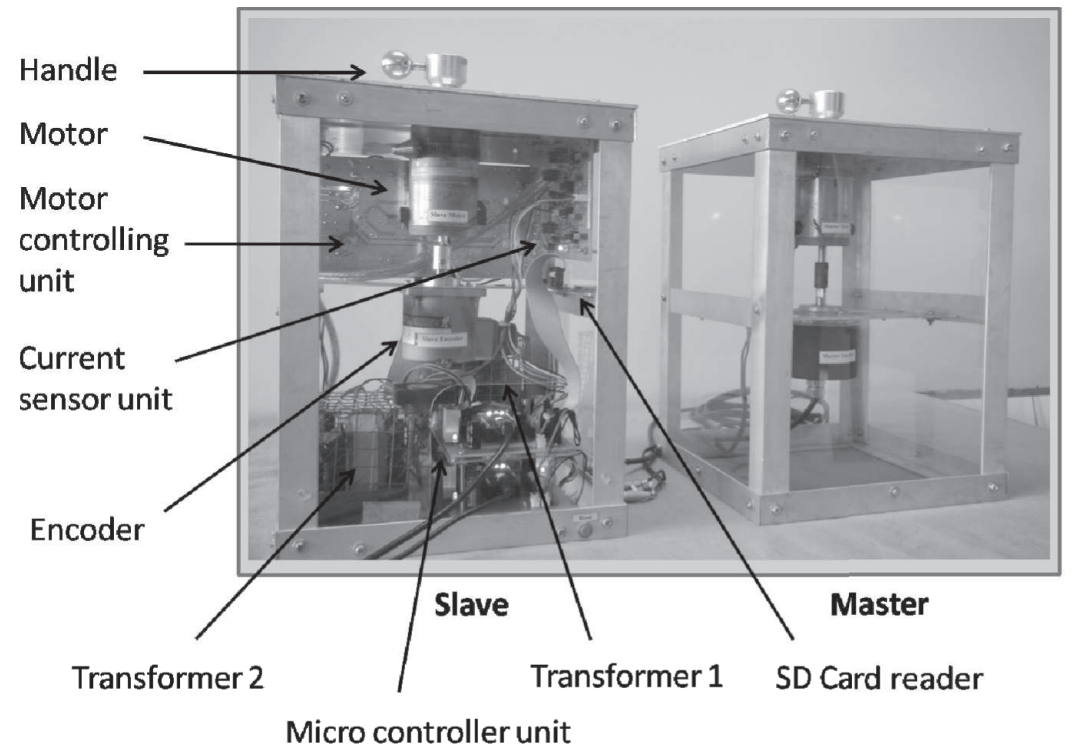

Figure 8: Hardware experimental systems 


\section{CONSTRUCTION}

\section{Hardware}

The bilateral control functionality was experimented on a hardware setup consisting of master and slave manipulator units. Two similar DC motors (by Electrocraft Inc.) were used for each unit. Figure 8 shows the arrangement of the hardware experimental platform.
The hardware platform consists of two PWM driven motor drivers with two driver ICs (DRV8432 by Texas Instrument), which can carry a current up to $14 \mathrm{~A}$ with a 24 A peak load. The motor drivers are operated by the PWM signals generated from mbed NXPLPC1768 microcontroller. Position sensing is done by two encoders coupled with master and slave manipulators, having $5000 \mathrm{ppr}$ and $2500 \mathrm{ppr}$, respectively. The hardware functional block diagram of the above system is shown in Figure 9.

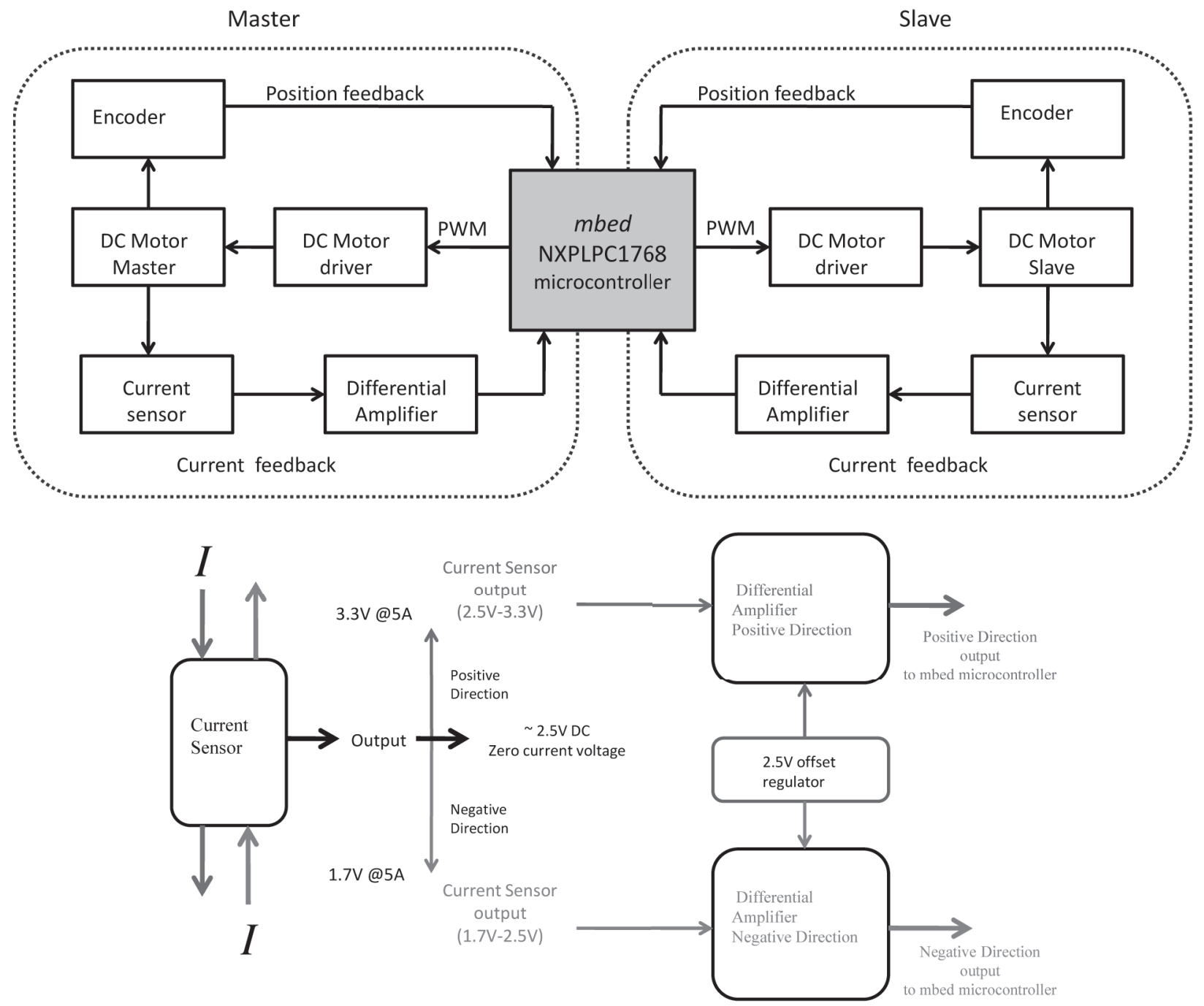

Figure 9: Hardware functional block with current sensor module

\section{Current sensor module operation}

The current sensor module comprises two identical current sensors (ACS712t by Allegro Microsystems
Inc.). Figure 9 shows the functionality of a current sensor, which also senses a current up to $5 \mathrm{~A}$ in both directions as shown in the diagram. It also shows the current flowing direction through the current sensor; The blue colour 
direction taken as the positive direction while the red colour direction taken as negative direction. When current flows in the positive direction, the output increases from "zero current voltage" set at 2.5 VDC, with a gradient of $185 \mathrm{mV} / \mathrm{A}$. Therefore, approximately $3.3 \mathrm{~V}$ will appear at the current sensor output when current is $5 \mathrm{~A}$ in the positive direction. The voltage difference of $0.8 \mathrm{~V}$ is fed to a differential amplifier, which has an amplified output range from $0-3.3 \mathrm{~V}$. The negative direction operation of the current sensor is also the same as that of the positive direction.

For a $5 \mathrm{~V}$ supply voltage, when there is no current flowing through the sensor, the voltage output for the zero current is $\sim 2.5 \mathrm{~V}$. The output of the current sensor varies positively or negatively around $2.5 \mathrm{~V}$ (from 1.7 $-3.3 \mathrm{~V}$ ) depending on the direction of the current flow. This output is fed into high impedance input, differential amplifier to amplify with respect to the direction of current flows. The differential amplifiers have high impedance inputs and there is no significant voltage drop and current consumption at the sensor output.

Each current sensor output is fed into two differential amplifiers. There are four differential amplifiers in the system. Two differential amplifiers attached to a motor (current sensor) are responsible to amplify the current flowing in the current sensor separately in either direction. Normally, the "zero current voltage" ( 2.5 VDC) is applied to the reference terminal, while the output from the current sensor is fed into the input terminal of the differential amplifier. The net input to the differential amplifier is about $0.8 \mathrm{~V}$ and it amplifies up to $3.3 \mathrm{~V}$ before feeding into the microcontroller. The reference supply is always maintained constantly throughout the operation.

\section{Software}

The mbed NXPLPC1768 microcontroller used in this research has limited resources. In order to optimize the resources of the microcontroller, a real time operating system (RTOS) is incorporated. The motor torques are determined by measuring the current through the motors. The outputs of two current sensors are amplified to obtain a $0-3.3 \mathrm{~V}$ signal. Two directions of rotation for two motors give four current output signals, which are fed into four analog input pins of the microcontroller.

\section{RESULTS AND DISCUSSION}

The controller implemented in the microcontroller was evaluated by an experiment with actual master-slave manipulators. In this experiment, the master manipulator is operated by a human, where the slave is in an unknown
Table 1: DC motor specifications

\begin{tabular}{lcc}
\hline Parameter & Value & Unit \\
\hline Rated output & 0.2 & $\mathrm{~kW}$ \\
$\begin{array}{l}\text { Rated/maximum torque } \\
\text { Encoder resolution }\end{array}$ & $20.5 / 169.5$ & $\mathrm{~N} \mathrm{~cm}$ \\
$\begin{array}{l}\text { Master } \\
\text { Slave }\end{array}$ & 5000 & Pulses/rev \\
\hline
\end{tabular}

Table 2: Experimental parameters

\begin{tabular}{llcc}
\hline Parameters & Symbol & Value & Units \\
\hline Motor inertia & $J_{n}$ & 0.268 & $\mathrm{kgcm}^{2}$ \\
Torque coefficient & $K_{t}$ & 13.5 & $\mathrm{Ncm} / \mathrm{Amp}$ \\
Proportional constant & $K_{p}$ & 0.01 & $\mathrm{rad} / \mathrm{sec}$ \\
Integral constant & $K_{i}$ & 0.0 & $\mathrm{rad} / \mathrm{sec}$ \\
Derivative constant & $K_{d}$ & 0.001 & $\mathrm{rad} / \mathrm{sec}$ \\
Cut-off frequency low & $K_{d i s}$ & $0-200$ & $\mathrm{~Hz}$ \\
pass filter. & & & \\
\hline
\end{tabular}

environment. Master and slave manipulators are controlled by the bilateral controller.

All computations are written in $\mathrm{C}$ language under real time operating system (RTOS) with a sampling time of $150 \mu \mathrm{s}$. Time critical main control programme was invoked at every $150 \mu$ s, while the data writing task is given the lowest priority. The specifications of the motors are given in Table 1 and the parameters used in the experiment are shown in Table 2.

The experiment was conducted for non-contact and contact motions of the bilateral control system. In this setup, the system was operated with a force bandwidth of 0 - $30 \mathrm{~Hz}$ (observer gain: 0-200 rad/s). The experiments were conducted with two different observer gains: $g_{d i s}=$ $50 \mathrm{rad} / \mathrm{s}$ and $g_{\text {dis }}=200 \mathrm{rad} / \mathrm{s}$.

In this experimental setup, there are two main units: master and slave manipulators. The master side was operated by human interaction. In an ideal bilateral tele-operation, the position and the torque applied at the master manipulator should appear as it is in the slave manipulator. In this test, slave side manipulator was not in contact with any material. Thus it was freely movable.

In the contact motion test, the slave unit is placed in an environment where the manipulator movement comes in contact with an object (nylon foam). Figures 10-13 show the experimental results of position and torque responses in both contact and non-contact motion. 


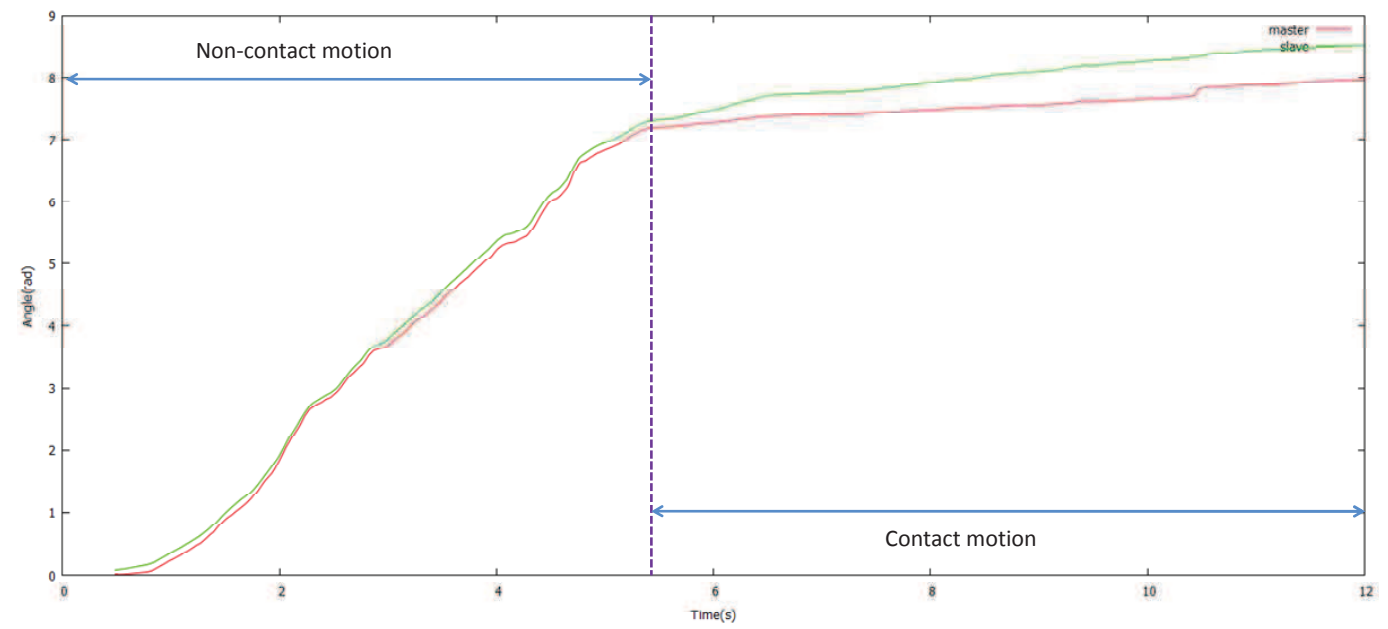

Figure 10: Position response, $g_{\text {dis }}=50 \mathrm{rad} / \mathrm{s}$

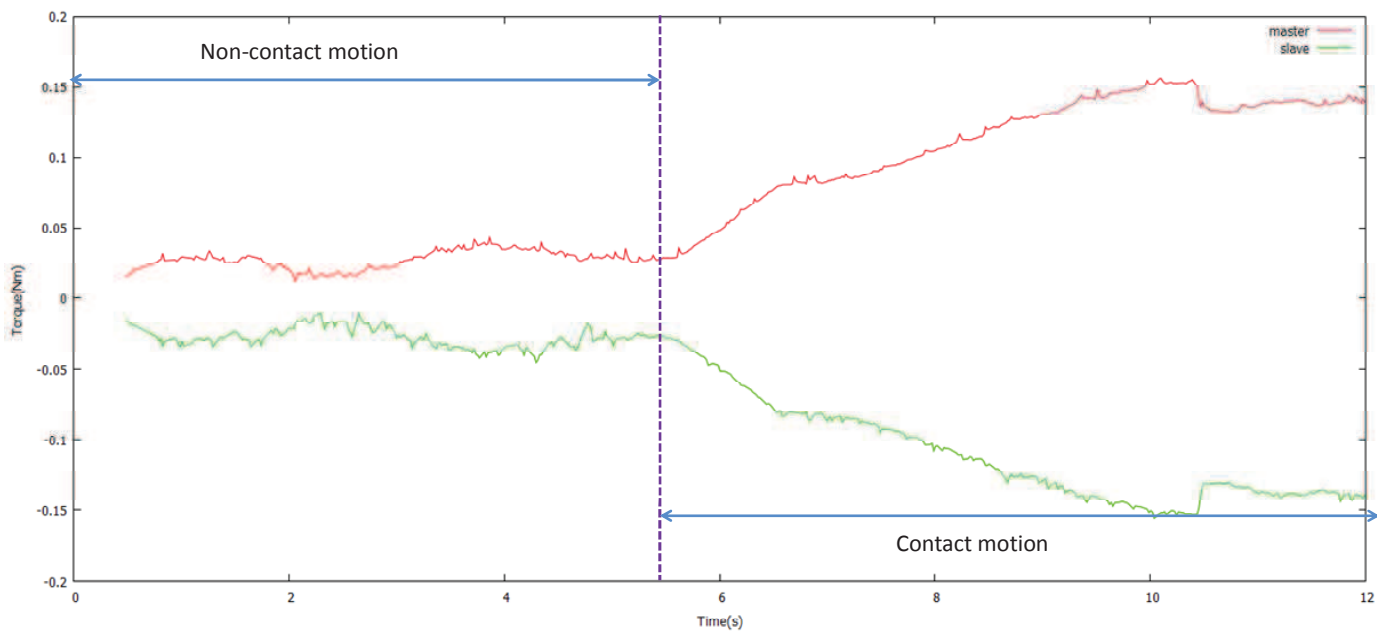

Figure 11: Torque response, $g_{d i s}=50 \mathrm{rad} / \mathrm{s}$

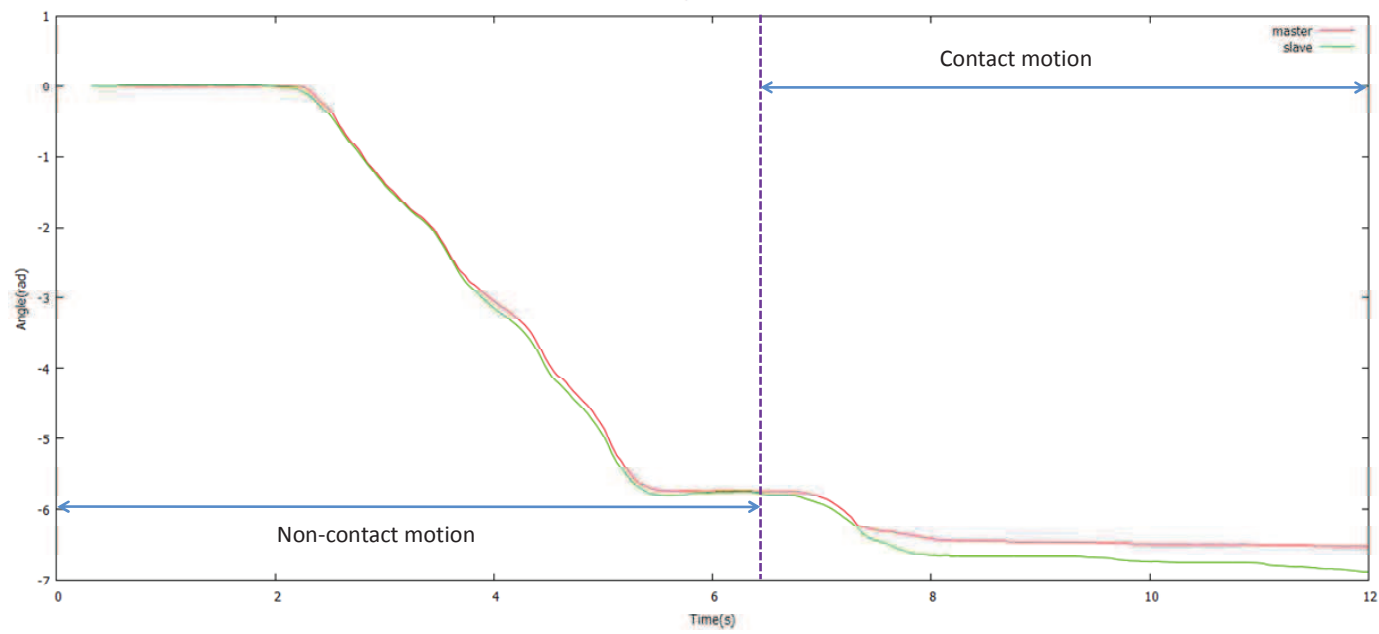

Figure 12: Position response, $g_{d i s}=200 \mathrm{rad} / \mathrm{s}$ 


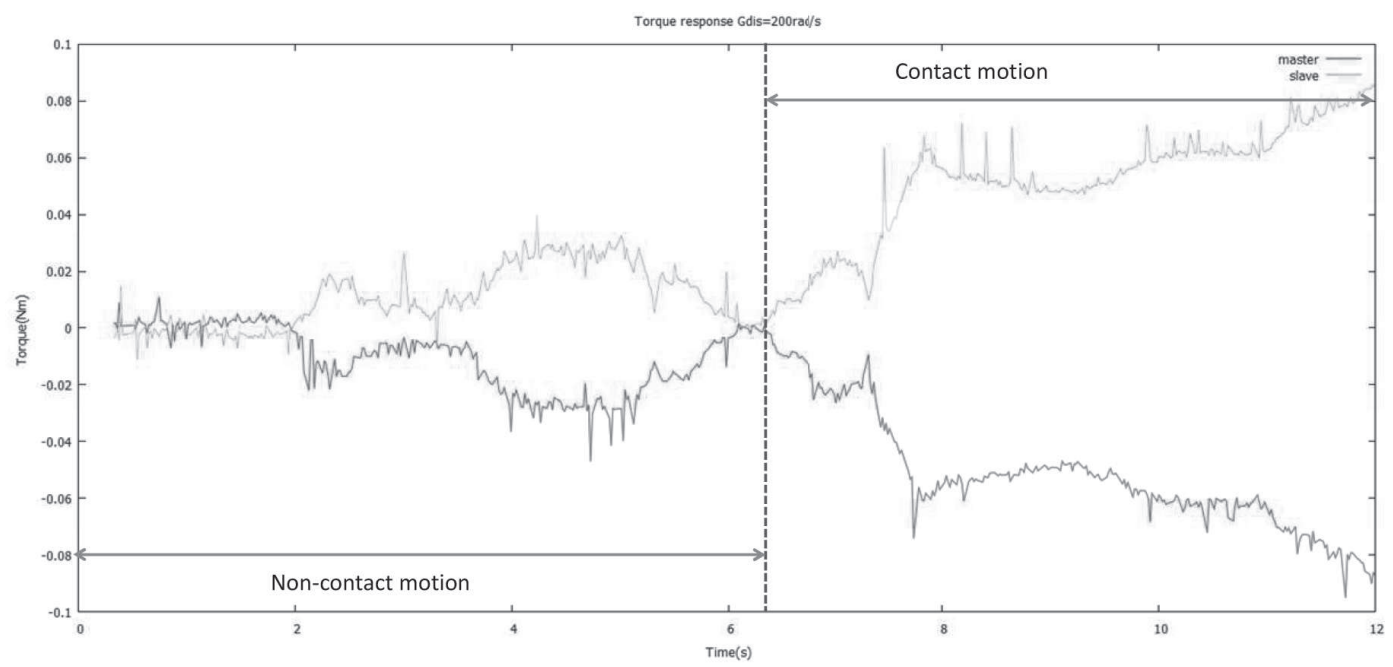

Figure 13: Torque response, $g_{\text {dis }}=200 \mathrm{rad} / \mathrm{s}$

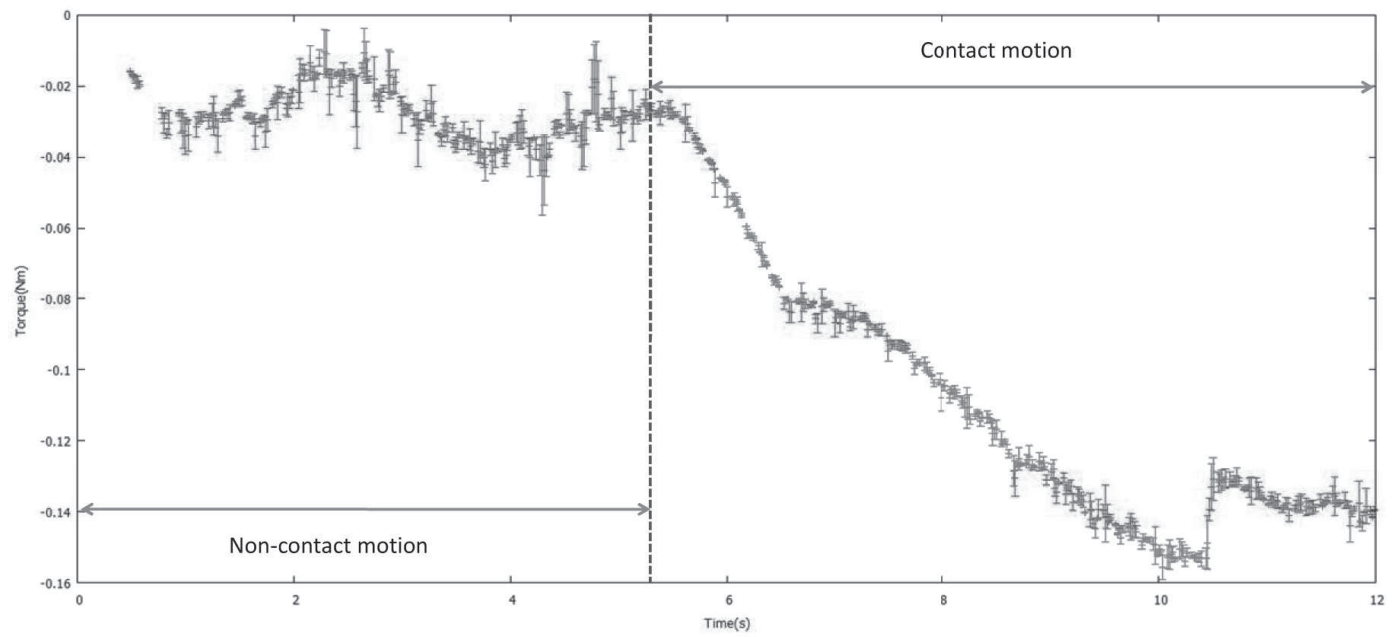

Figure 14: Torque error response, Gdis $=50 \mathrm{rad} / \mathrm{s}$

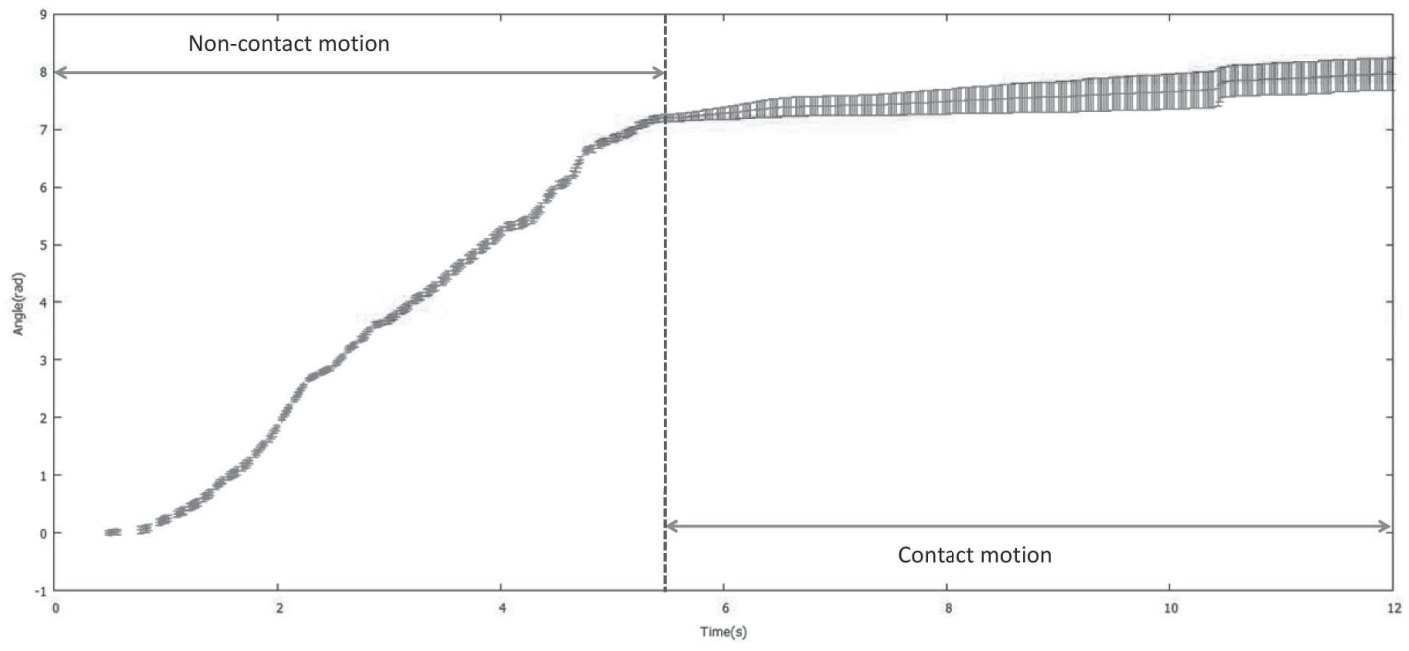

Figure 15: Position error response, Gdis $=50 \mathrm{rad} / \mathrm{s}$ 


\section{Non-contact motion test}

Figures 10 and 12, clearly shows that the position response of the slave manipulator is closely following the position response of the master manipulator. In both experiments (for different observer gains; $g_{\text {dis }}=50 \mathrm{rad} / \mathrm{s}$, $g_{\text {dis }}=200 \mathrm{rad} / \mathrm{s}$ ), position errors are insignificantly smaller as shown in Figure 15 (maximum position error is less than $0.6 \%$ ).

The torque responses are shown in Figures 11 and 13. The master and slave torques follow the same pattern; the mirror image of the other (as per equation 13). The response of the slave manipulator closely follows the torque applied at the master manipulator as shown in Figure 11. Figures 11 and 13 show the torque responses in different cut-off frequencies (DOB gain). A higher cutoff frequency (Figure 13) shows high frequency variations of the torque when compared to the Figure 11.

\section{Contact motion test}

Torque response of the slave manipulator in contact motion has followed the master manipulator as shown in Figures 11 and 13. Figure 14 shows the error bar generated on top of the "master" torque curve. As for the results, torque error is less than $20 \%$ in non-contact motion and it has reduced to a value less than $4 \%$ in the contact motion test.

Position response of the slave manipulator in contact motion has followed the master manipulator as shown in Figures 10 and 12. The result of the contact motion test shows a considerable error in position response. This was due to the low proportional gain selected in the controller. However, it could not be increased as for the torque limits of the motor. According to the error bar shown in Figure 15, the position response error is always less than $6 \%$.

\section{CONCLUSION}

In this research, the bilateral control system was realized by using a microcontroller in which the available resources in the processor were limited compared to other existing high speed processors. The operation bandwidth of the torque response was $0-30 \mathrm{~Hz}$. Position servoing and torque servoing were accomplished with the master-slave manipulator units separately for contact and non-contact motions. The position in the non-contact motion test very closely follows the master manipulator's position. Torque response in contact motion shows a lower error compared to the non-contact motion. This error could be reduced by incorporating high speed processors and maintaining a smaller sampling time. Sampling time was kept constant by using the microcontroller based RTOS. A motor driver system was developed with a current controller to meet the embedded system's requirements. This system can be specially used in embedded systems based bilateral controllers where the mobility is required.

\section{Acknowledgment}

The authors would like to express their gratitude to MBED (mbed.org) for providing microcontrollers for this research.

\section{REFERENCES}

1. Abeykoon A.M.H.S. (2008). Virtual impedance models for bilateral motion control. PhD thesis, Keio University, Japan.

2. Abeykoon A.M.H.S. \& Ohnishi K. (2006). Bilateral control interacting with a virtual model and environment. Proceedings of the IEEE conference on Industrial Technology, December 2006, Mumbai, India, pp. 1320 1325 .

3. Abeykoon A.M.H.S. \& Ohnishi K. (2007). Virtual tool for bilaterally controlled forceps robot-for minimally invasive surgery. Journal of Medical Robotics and Computer Assisted Surgery 3(3): $271-280$.

DOI: http://dx.doi.org/10.1002/rcs.147

4. Abeykoon A.M.H.S. \& Ohnishi K. (2008). Improvement of tactile sensation of a bilateral forceps robot by a switching. Virtual Model Journal of Advanced Robotics 8: 789 - 806. DOI: http://dx.doi.org/10.1163/156855308X314506

5. Abeykoon A.M.H.S. \& Senevirathne H.R. (2012). Disturbance observer based current controller for a brushed DC motor. Proceeding of the $6^{\text {th }}$ IEEE Conference on Information and Automation, September 2012, Beijing, China, pp. $59-64$.

6. George C., Lee S., Gonzalez R.C. \& Fu K.S. (1983). Tutorials on Robotics. IEEE Computer Society Press, Washington DC, USA.

7. Hashtrudi-Zaad K. \& Salcudean S.E. (2002a). Bilateral parallel force/position teleoperation control. Journal of Robotics Systems 19(4): 155 - 167.

DOI: http://dx.doi.org/10.1002/rob.10030

8. Hashtrudi-Zaad K. \& Salcudean S.E. (2002b). Transparency in time-delayed systems and the effect of local force feedback for transparent teleoperation. IEEE Transactions on Robotics and Automation 18(1): 108 - 114.

DOI: http://dx.doi.org/10.1109/70.988981

9. Itoh T., Kosuge K. \& Fukuda T. (2000). Human-machine cooperative telemanipulation with motion and force scaling using task-oriented virtual tool dynamics. IEEE Transaction on Robotics and Automation 16(5): 505 - 516.

DOI: http://dx.doi.org/10.1109/70.880801 
10. Katsura S. (2004). Advance motion control based quarry of environmental information. $P h D$ thesis, Keio University, Japan.

11. Katsura S., Irie K. \& Ohnishi K. (2008). Wideband force control by position-acceleration integrated disturbance observer. IEEE Transactions on Industrial Electronics 55(4): $1699-1706$.

DOI: http://dx.doi.org/10.1109/TIE.2007.907664

12. Katsura S., Matsumoto Y. \& Ohnishi K. (2006). Analysis and experimental validation of force bandwidth for force control. IEEE Transaction on Industrial Electronics 15(3): $922-928$.

DOI: http://dx.doi.org/10.1109/TIE.2006.874262

13. Katsura S., Suzuyama T. \& Ohnishi K. (2007). Bilateral teleoperation with different configuration using interaction mode control. Proceedings of the IEEE International Symposium on Industrial Electronics, June 2007, Vigo, Spain, pp. $3120-3125$.

DOI: http://dx.doi.org/10.1109/ISIE.2007.4375114

14. Lawrence D. (1993). Stability and transparency in bilateral teleoperation. IEEE Transactions on Robotics and Automation 9(5): $624-637$.

DOI: http://dx.doi.org/10.1109/70.258054

15. Mayer H., Nagy I., Knoll A., Schirmbeck E.U. \& Bauernschmitt R. (2005). An experimental system for robotic heart surgery. Proceedings of the $18^{\text {th }}$ IEEE International Conference on Computer-Based Medical Systems, June 2005, Dublin, Ireland, pp. 23 - 24.

16. Murakami T.Y.U.F. \& Ohnishi K. (1993). Torque sensorless control in multidegree-of-freedom manipulator. IEEE Transactions on Industrial Electronics 40(2): 259 - 265.

17. Nishimura K., Shimono T. \& Ohnishi K. (2006). Improvement of reproducibility and operationality for bilateral control by nominal mass design. Proceedings of the IEEE conference on Industrial Technology, December 2006, Mumbai, India, pp. 1326 - 1331. DOI: http://dx.doi.org/10.1109/ICIT.2006.372540

18. Ohishi K., Nakao M. \& Ohnishi K. (1987). Microprocessor - controlled DC motor for load-insensitive position servo system. IEEE Transactions on Industrial Electronics 34(1): $44-49$.

DOI: http://dx.doi.org/10.1109/TIE.1987.350923
19. Ohnishi K., Matsui N. \& Hori Y. (1994). Estimation identification and sensorless control in motion control system. Proceedings of the IEEE conference on Industrial Technology, December 1994, Tokyo, Japan, pp. 1253 1265.

20. Ohnishi K., Shibata M. \& Murakami T. (1996). Motion control for advanced mechatronics. IEEE/ASME Transactions on Mechatronics 1(1): 56 - 67.

21. Sabanovic A. (2003). Sliding modes in power electronics and motion control systems. Proceedings of the $29^{\text {th }}$ IEEE Annual Conference of the Industrial Electronics Society, November 2003, Virginia, USA, pp. $997-1002$.

22. Senevirathne H.R., Abeykoon A.M.H.S. \& Pillai M.B. (2012). Disturbance rejection analysis of a disturbance observer based velocity controller, Proceeding of the 6th IEEE Conference ICIAfS'12, September 2012, Beijing, China, pp. $59-64$.

23. Shimono T. (2007). Advance motion control for real world haptic storage. PhD thesis, Keio University, Japan.

24. Shimono T., Katsura S. \& Ohnishi K. (2005). Improvement of operationality for bilateral control based on nominal mass design in disturbance observer. Proceeding of the $31^{\text {st }}$ Annual IEEE Conference on Industrial Electronics, November 2005, North California, USA, pp. 229 - 233.

25. Sumiyoshi Y. \& Ohnishi K. (2004). The transformation of modified 4-channel architecture. $8^{\text {th }}$ IEEE International workshop on Advance Motion Control, March 2004, Kawasaki, Japan, pp. $211-216$.

26. Takeo K. \& Kosuge K. (1997). Implementation of the micromacro teleoperation system without using slave-side force sensors. Proceeding of the IEEE International Conference on Robotics and Automation, April 1997, Albuquerque, New Mexico, pp. 20 - 25.

27. Tsuji T. \& Ohnishi K. (2004). Position/force scaling of function based bilateral control system. Proceeding of the IEEE International Conference on Industrial Technology, December 2004, Hammamet, Tunisia, pp. 96 - 101.

28. Yokokohji Y. \& Yoshikawa T. (1994). Bilateral control of master-slave manipulator for ideal kinesthetic couplingformulation and experiment. IEEE Transaction on Robotics and Automation 10(5): $605-620$. DOI: http://dx.doi.org/10.1109/70.326566 